\title{
Impurity transport due to electromagnetic drift wave turbulence
}

\author{
S. Moradi, I. Pusztai, A. Mollén, and T. Fülöp \\ Department of Applied Physics, Nuclear Engineering, Chalmers University of Technology and Euratom-VR \\ Association, Göteborg, Sweden
}

(Received 2 November 2011; accepted 26 January 2012; published online 5 March 2012)

\begin{abstract}
Finite $\beta$ effects on impurity transport are studied through local linear gyrokinetic simulations with GYRO [J. Candy and E. Belli, General Atomics Report No. GA-A26818, 2011]; in particular, we investigate the parametric dependences of the impurity peaking factor (zero-flux density gradient) and the onset of the kinetic ballooning modes (KBMs). We find that electromagnetic effects even at low $\beta$ can have significant impact on the impurity transport. The KBM instability threshold depends on the plasma parameters, particularly strongly on plasma shape. We have shown that magnetic geometry significantly influences the results, and the commonly used $s$ - $\alpha$ model overestimates the KBM growth rates and ITG stabilization at high $\beta$. In the $\beta$ range, where the KBM is the dominant instability the impurity peaking factor is strongly reduced, with very little dependence on $\beta$ and the impurity charge. (C) 2012 American Institute of Physics.

[http://dx.doi.org/10.1063/1.3688876]
\end{abstract}

\section{INTRODUCTION}

Impurity transport is a critical issue in burning plasma regimes due to possible fuel dilution and radiative cooling in the core. Impurity transport due to electrostatic microinstabilities has been widely studied in previous works; however, the impact of electromagnetic effects at finite $\beta$ on the transport of impurities has not received as much attention. Here $\beta=8 \pi\langle p\rangle / B^{2}$, where $\langle p\rangle$ is the volume average kinetic pressure and $B$ is the toroidal magnetic field. In the view of an increasing interest in high $\beta$ operation scenarios, such as hybrid scenarios for ITER (Refs. 1, 2), the question of finite $\beta$ effects on the impurity transport deserves further investigation.

Previous works have reported $\beta$ scaling of the eigenfrequency and turbulence mode structures both with gyro-fluid ${ }^{3}$ as well as gyrokinetic ${ }^{4-10}$ models. It has been found that electromagnetic effects have stabilizing influence on the ion temperature gradient (ITG) modes. However, at finite ion temperature gradient and below the ideal magnetohydrodynamic (MHD) ballooning mode threshold, $\beta<\beta_{M H D}$, a branch of kinetic ballooning modes (KBMs) (in some older work, the name AITG has been used to refer to the KBM [Ref. 11]) can become destabilized. It has been shown that the ITG modes, driven by the $\nabla B$-curvature and ion pressure gradient, are essentially an electrostatic instability, whereas KBMs are electromagnetic instabilities due to the perpendicular interchange dynamics coupling to parallel Alfvén wave dynamics.

From the experimental side, effects of finite $\beta$ on the transport of light impurities such as helium and tritium have been explored, and a reduction in diffusivities of the light impurities as $\beta$ increases has been reported. ${ }^{12,13}$ Theoretical studies have confirmed these observations qualitatively, and it has been shown that an increase in $\beta$ will result in a stabilization of the ITG modes leading to significant reduction of the impurity diffusivities. ${ }^{14}$ However, this study shows that for low impurity charges, the ratio of the convective velocity to the diffusivity (peaking factor) is not strongly affected by an increase in $\beta$, while it increases in magnitude with increasing $\beta$ for heavy impurities. Therefore, an increase in $\beta$ is predicted to cause peaking of the heavy impurity profiles. It has to be noted that in this study, no KBMs were present for the whole range of $\beta$ considered. However, recent metal impurity transport control experiments in JET H-mode plasmas with $\beta_{e} \gtrsim 0.004$ (at the inner mid-radius) have shown much lower peaking of nickel impurity in the plasma core (normalized radius $\rho<0.5$ ) than further out; in particular, a sign change of the peaking factor (zero-flux density gradient) from positive to negative has been observed for inner radii. ${ }^{15}$ Here, the effective normalized electron pressure is defined as $\beta_{e}=8 \pi n_{e} T_{e} / B^{2}$; note that for $T_{i}=T_{e}$, we have $\beta=2 \beta_{e}$. These results could not be explained within the framework of an electrostatic transport model, since the most unstable modes under these conditions are predicted to be ITG modes, and impurity driven transport due to electrostatic ITG modes always leads to positive peaking factors. In the present paper, we investigate the impact of finite $\beta$ effects on the transport of impurities. Our study concludes, however, that at the experimentally relevant $\beta$, comparable peaking factors are expected from midradius towards the core.

The onset of the KBM instability is also studied. KBMs propagate in the ion diamagnetic direction and can be destabilized above a critical value of $\beta_{e}$ (which we denote by $\beta_{e}^{\text {crit }}$ ). We find that for typical experimental parameters, $\beta_{e}$ is well below $\beta_{e}^{\text {crit }}$ in the core of tokamaks; however, electromagnetic effects even at low $\beta_{e}$ can have significant impact on the impurity transport, and an electrostatic treatment in the core can give misleading results. The quasilinear impurity transport is computed using the gyrokinetic code GYRO ${ }^{16,17}$ All the simulations are performed in the flux-tube (local) limit. Our results show that for the considered plasma parameters, two possible modes can be unstable depending on the normalized electron pressure; ITG modes dominate in the region $\beta_{e} \lesssim 0.015$, whereas KBM dominates for $\beta_{e} \gtrsim 0.015$. 
Consistently with previous studies, we also observe the stabilization of ITG modes by electromagnetic effects as $\beta_{e}$ increases. For the experimentally relevant parameters used in our study, the KBM stability threshold $\beta_{e}^{\text {crit }}$ is about a factor three higher than the experimental value of $\beta_{e}$. The sensitivity of this threshold on the various plasma parameters has been analyzed here, and it is found that it is highly sensitive to the plasma shape and magnetic geometry; however, other effects such as collisions and the gradients of density and temperatures may also lead to significant change in the KBM threshold. The importance of plasma shape on the onset of the KBM instability and plasma confinement properties was reported in Ref. 18, where the results from a series of $\beta$ scaling experiments performed at ASDEX-U and DIII-D tokamaks are discussed. In Ref. 18, gyrokinetic analysis with GYRO code is presented, and it is shown that as $\beta_{e}$ is increased, the most unstable mode changes from ITG at low $\beta_{e}$ to microtearing at intermediate values of $\beta_{e}$ and to $\mathrm{KBM}$ at high values of $\beta_{e}$. In the KBM branch, they observe significantly higher growth rates increasing strongly with $\beta_{e}$ in comparison to the ITG and micro-tearing branches. In the present work, however, no micro-tearing mode is observed for the whole range of $\beta_{e}$ considered, and more interestingly, the growth rates of the KBM is comparable to that of the ITG mode with no strong increase with increase of $\beta_{e}$.

The $\beta_{e}$ scaling of the impurity peaking factor shows two branches in connection with the two branches of the unstable modes present. The peaking factor increases with $\beta_{e}$ in the ITG branch, but it exhibits only a very weak $\beta_{e}$ dependence in the KBM branch. The peaking factor values in the KBM branch are significantly lower, about half of those in the ITG branch, with negligible charge dependence. In the ITG branch, stronger dependence on the impurity charge is expected and this dependence increases as $\beta_{e}$ increases; however, for heavy impurities with lower charge to mass ratio such as tungsten, lower peaking factors with very little $\beta_{e}$ dependence is observed.

The remainder of the paper is organized as follows. In Sec. II, the model for calculating the quasilinear impurity flux and the peaking factor is presented. In Sec. III, the parametric dependences of the peaking factor are analyzed by presenting scans over relevant parameters such as charge number and temperature and density scale lengths. Also, the scans for magnetic shear, safety factor, and collisions are demonstrated. Finally, the results are discussed and summarized in Sec. IV.

\section{IMPURITY FLUX AND PEAKING FACTOR}

The quasilinear impurity particle flux is given by

$$
\begin{aligned}
\Gamma_{z}= & -\frac{k_{\theta}}{B} \Im\left[\int d ^ { 3 } v g _ { z } \left(J_{0}\left(z_{z}\right) \phi^{*}-J_{0}\left(z_{z}\right) v_{\|} \frac{\delta A_{\|}^{*}}{c}\right.\right. \\
& \left.\left.+\frac{v_{\perp}}{k_{\perp}} J_{1}\left(z_{z}\right) \frac{\delta B_{\|}^{*}}{c}\right)\right],
\end{aligned}
$$

where $\Im[\cdot]$ denotes imaginary part, $k_{\theta}$ is the poloidal wave-number, $B$ is the equilibrium magnetic field, $g_{z}$ is the non-adiabatic part of the perturbed impurity distribution
TABLE I. Input parameters for densities, temperatures and their gradients.

\begin{tabular}{cccccccccc}
\hline \hline & $\rho_{\text {tor }}$ & $r$ & $n_{e}\left[10^{19} / \mathrm{m}^{3}\right]$ & $T_{e}[\mathrm{keV}]$ & $a / L_{n e}$ & $a / L_{T i}$ & $a / L_{T e}$ & $T_{i} / T_{e}$ & $\rho_{s} / a$ \\
\hline case 1 & 0.3 & 0.347 & 3.0 & 7.0 & 1.5 & 2.5 & 2.0 & 0.85 & 0.0035 \\
case 2 & 0.5 & 0.555 & 2.5 & 3.8 & 1.0 & 3.0 & 1.5 & 0.84 & 0.0028 \\
\hline \hline
\end{tabular}

function, $J_{0}$ and $J_{1}$ are the Bessel functions of the first kind, $z_{z}=k_{\perp} v_{\perp} / \omega_{c z}, k_{\perp}=k_{\theta} \sqrt{1+s^{2} \theta^{2}}, s$ is the magnetic shear, $\theta$ is the ballooning angle, $\omega_{c z}=Z e B /\left(c m_{z}\right)$ is the cyclotron frequency, and $\phi^{*}, \delta A_{\|}^{*}$, and $\delta B_{\|}^{*}$ are the complex conjugates of the perturbed electrostatic potential and the transverse and compressional magnetic perturbations, respectively.

In order to find the impurity peaking factor, we make a scan in $a / L_{n z}$ at a fixed $a / L_{n e}$ and vary $a / L_{n i}$ in order to satisfy the quasi-neutrality condition. Using an interpolation scheme, we then search for the $a / L_{n z}$ corresponding to zero flux. Here, $L_{n a}=-\left[\partial\left(\ln n_{a}\right) / \partial r\right]^{-1}$ and $L_{T a}=-\left[\partial\left(\ln T_{a}\right) / \partial r\right]^{-1}$ are the density and temperature scale lengths and $a$ is the outermost minor radius.

In the calculations presented in this section, we have used two sets of local profile parameters given in Table I.

Here, $\rho_{\text {tor }}$ is defined as

$$
\rho_{\text {tor }}=\sqrt{\chi_{t}(r) / \chi_{t}(a)}
$$

where $2 \pi \chi_{t}$ is the toroidal flux, and $\rho_{s}$ is the ion sound Larmor radius. To take into account the plasma shape, we have used a Miller-type local equilibrium model available in GYRO, see Refs. 19 and 20. Typical JET parameters for plasma shape and magnetic geometry are used, and the corresponding values are given in Table II.

In this table, $\beta_{e}$ is calculated in CGS units following the expression:

$$
\beta_{e}=\frac{8 \pi\left(n_{e}\left[10^{19} / m^{3}\right] 10^{-6} 10^{19}\right)\left(T_{e}[k e V] 1.602210^{-9}\right)}{\left(10^{4} B_{\text {unit }}[T]\right)^{2}},
$$

where $B_{\text {unit }}$ is defined as the effective field strength, see Refs. 19 and 20 ,

$$
B_{\text {unit }}=\frac{1}{r} \frac{d \chi_{t}}{d r}
$$

The safety factor is $q=d \chi_{t} / d \psi$, with $2 \pi \psi$ being the poloidal flux, and the magnetic shear is $s=(r / q) d q / d r$. Furthermore, $\varepsilon$ is the inverse aspect ratio, $\kappa$ is the elongation, $\delta$ is the triangularity, $\zeta$ is flux surface squareness, and $Z_{m a g}$ is the elevation of the flux surface. The convention of defining $\beta_{e}$ in terms of $B_{\text {unit }}$ is specific to GYRO and not to gyrokinetic codes in general. We note that $B_{\text {unit }}$ in shaped plasmas is not equal to the on-axis magnetic field $\bar{B}$, because the effective

TABLE II. Input parameters for plasma shape and magnetic geometry.

\begin{tabular}{rccccccccr}
\hline \hline & $\beta_{e}$ & $1 / \varepsilon$ & $B_{\text {unit }}[\mathrm{T}]$ & $s$ & $q$ & $\kappa$ & $\delta$ & $\zeta$ & $Z_{\text {mag }}$ \\
\hline case 1 & 0.0047 & 3.36 & 4.22 & 0.56 & 1.29 & 1.31 & 0.024 & 0.0053 & 0.22 \\
case 2 & 0.0016 & 3.34 & 4.68 & 1.06 & 1.91 & 1.36 & 0.058 & -0.0023 & 0.21 \\
\hline
\end{tabular}


radius $r$ is defined as the half width of the flux surface at the elevation of its centroid, and thus the toroidal flux inside a given flux surface is generally not equal to $r^{2} \pi \bar{B}$. Since for elongated flux surfaces $(\kappa>1)$, the area of the cross section of the flux surface is larger than $\pi r^{2}$ and the effective field is roughly $\kappa$ times larger than the on-axis field. It is important that it is $\beta_{e}$, defined with the effective field, that matters for the stability of KBMs, and it is reduced by approximately a factor of $1 / \kappa^{2}$ compared to a $\beta_{e}$ defined with $\bar{B}$. This should be taken into account when the experimental $\beta_{e}$ is compared to gyrokinetic simulations.

The normalized poloidal wave number is fixed to $k_{\theta} \rho_{s}=0.3$. These are the baseline cases in our study, and these parameters will be used unless otherwise stated. The impurities are assumed to be present in trace quantities, in the sense that $Z n_{z} / n_{e} \ll 1\left(n_{z} / n_{e}=2 \times 10^{-3}\right.$ is used in the simulations). These cases are taken from nickel laser blow off experiments at JET, featuring the injection of Radio Frequency (RF) power at ITER relevant collisionalities $\left(\nu_{\text {eff }}<0.2\right)\left(\right.$ shot number 68383). ${ }^{15}$

Figure 1 shows the growth rates and real frequencies of the unstable modes for the baseline parameters of case 1 . These results are obtained using the eigenvalue solver method in the GYRO code. ${ }^{17}$ As can be seen in this figure, the ITG mode is the most unstable mode for $\beta_{e}<0.015$ with a subdominant KBM present for the interval of $0.01<\beta_{e}<0.015$. For $\beta_{e}>0.015$, the most unstable mode present is the KBM and the ITG mode coexists as a subdominant mode having positive growth rate even at $\beta_{e}=0.02$. No trapped electron (TE) mode is unstable, in this case, for the whole range of $\beta_{e}$ considered. The real frequency of the KBM branch is significantly higher than that of the ITG branch; however, the maximal growth rates are comparable for the two modes.

To demonstrate how important the proper choice of the model geometry is in our analysis, we have considered a test case using a model based on $s-\alpha$ geometry available in GYRO. The results are shown in Fig. 2, which shows the growth rates

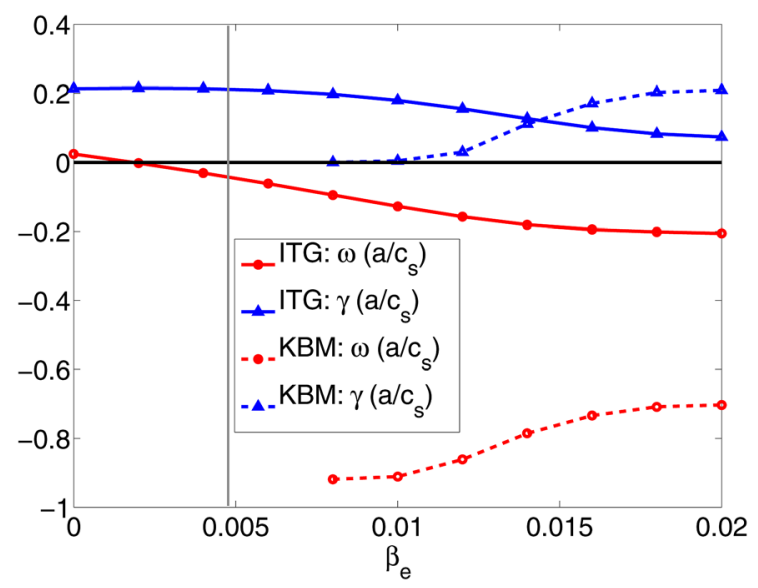

FIG. 1. (Color online) Real and imaginary parts of the eigenfrequency $\omega+i \gamma$ as function of $\beta_{e}$ obtained using the eigenvalue solver method in GYRO for the baseline case 1 using a model based on the Miller-type local equilibrium model. Red lines (with circle markers) represent the real part; blue lines (triangle markers) correspond to the imaginary part of the eigenvalue. Solid lines correspond to the ITG branch and dashed lines represent the KBM branch. The frequencies are normalized to $c_{s} / a$. The vertical line represents the experimental value of $\beta_{e}$.

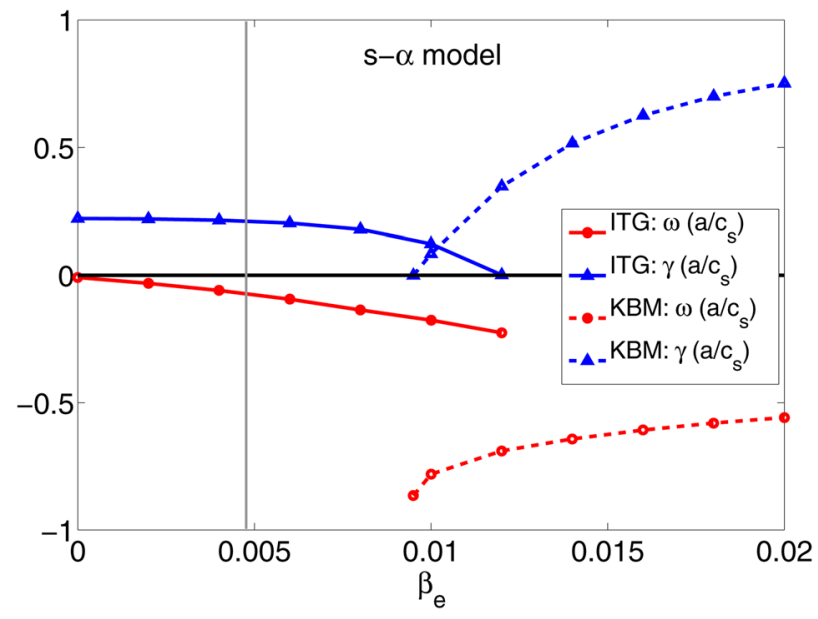

FIG. 2. (Color online) Real and imaginary parts of the eigenfrequency $\omega+i \gamma$ as function of $\beta_{e}$ obtained using the eigenvalue solver method in GYRO for the baseline case 1 using a model based on $s$ - $\alpha$ geometry. Red lines (with circle markers) represent the real part; blue lines (triangle markers) correspond to the imaginary part of the eigenvalue. Solid lines correspond to the ITG branch and dashed lines represent the KBM branch. The frequencies are normalized to $c_{s} / a$. The vertical line represents the experimental value of $\beta_{e}$.

and real frequencies of the unstable modes for the baseline parameters of case 1 . Here, the so called generalized magnetohydrodynamic $\alpha$ parameter is defined as (see Ref. 20)

$$
\alpha_{p}=-q^{2} R_{0} \frac{8 \pi}{B_{\text {unit }}^{2}} \frac{d p}{d r} c_{p},
$$

where $R_{0}$ is the effective major radius, $r$ is the minor radius, and $p=\sum_{a} n_{a} T_{a}$ is the total plasma pressure. $c_{p}$ is the geometric pressure gradient scaling parameter which allows an artificial adjustment of $\alpha_{p}$ without modifying the background gradients as presented in Ref. 7 . The connection between the two geometry models: Miller-type local equilibrium and $s-\alpha$ geometry are explained in details in Ref. 20. In short, $s-\alpha$ model can be recovered from a Miller-type local equilibrium by taking the limit $r / R_{0} \rightarrow 0$. As seen in Fig. 2, the results are significantly different between the two models. With the $s$ - $\alpha$ model, the onset of the KBM has not changed, but its growth rate is now significantly higher than that of the ITG mode and it is strongly increasing with $\beta_{e}$. Also, the ITG mode is completely stabilized for $\beta_{e}>0.012$, whilst it still coexists as a subdominant mode with a Miller-type model as shown in Fig. 1. Since the $s-\alpha$ model is less accurate in defining the plasma shape, and as it is shown later that plasma shape and magnetic geometry have significant impact on the results, in the remainder of the present paper, we only use the Miller-type model.

Even though the results shown in Fig. 1 indicate that two branches of unstable modes can coexist in a certain range of $\beta_{e}$, in the following, we only consider the most unstable mode for calculating the impurity peaking factor. We adopted this simplification, because linear theory does not provide the absolute magnitude of the perturbed quantities, and accordingly, the fluxes driven by the different branches cannot be compared (or simply added together). This approximation is expected to be valid in the limits where the ratio of the ITG and TE mode growth rates is not close to unity. 

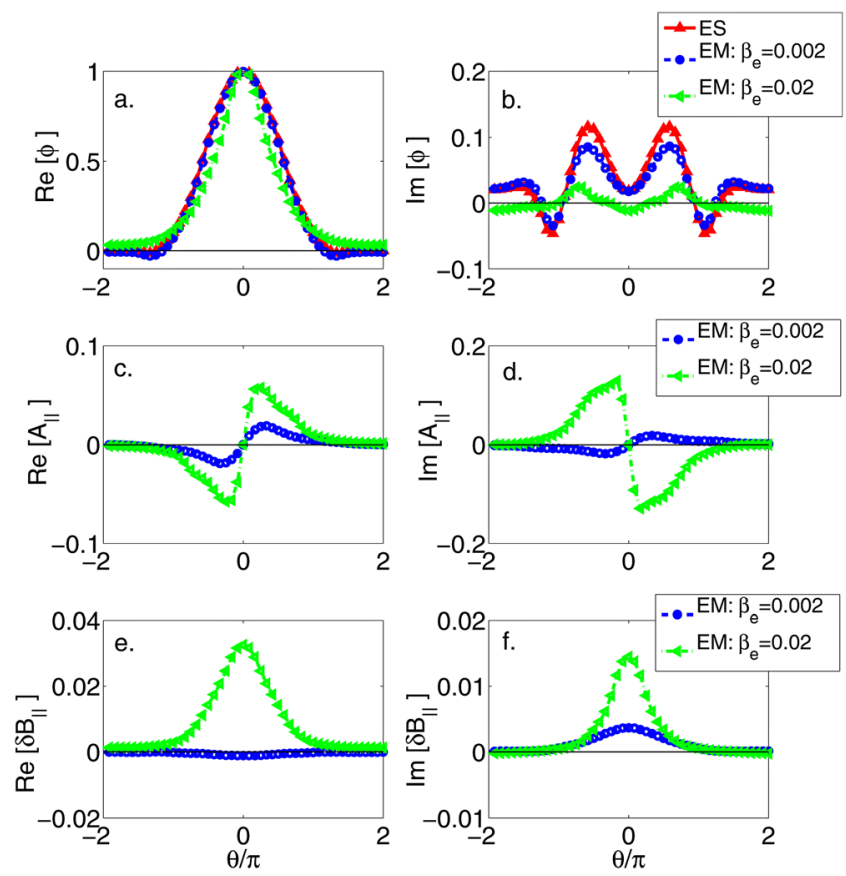

FIG. 3. (Color online) (a) Real and (b) imaginary parts of the perturbed electrostatic potential $\phi$ as a function of normalized ballooning angle for the plasma parameters of the baseline case 1. (c), (e) Real and (d), (f) imaginary parts of the transverse $A_{\|}$and compressional $\delta B_{\|}$magnetic perturbations, respectively. Red lines (up-triangle markers) show the electrostatic (ES) case, blue lines (circle markers) show the electromagnetic (EM) case with $\beta_{e}=0.002$ (ITG branch), and green lines (left-triangle markers) show the EM case with $\beta_{e}=0.02$ (KBM branch). The radial resolution of the simulations corresponds to a coverage in extended poloidal angle over the range $\theta / \pi=(-16,16)$, however to assist visibility a smaller range of $\theta$ is plotted.

Figure 3 shows the structure of the eigenmodes for the baseline case 1 and different values of $\beta_{e}$. The two branches of ITG (at $\beta_{e}=0.002$ ) and KBM (at $\beta_{e}=0.02$ ) can be distinguished by comparing the $\delta A_{\|}$in Figs. 3(c) and 3(d), where the imaginary and real components are in-phase for the ITG branch and out-of-phase in the KBM branch (so called KBM symmetry ${ }^{7}$ ).

\section{PARAMETRIC DEPENDENCES OF THE PEAKING FACTOR}

Figures 4(a), 4(b), and 4(c) show the growth rates and real frequencies of the most unstable modes and the peaking factors for nickel as a function of $\beta_{e}$ for the baseline parameters of cases 1 and 2 . These results are obtained using the linear initial value solver mode of GYRO. In Fig. 4(a), the solid lines correspond to case 1 and are similar to those in Fig. 1, which were obtained using the eigenvalue solver mode. Case 2 (plotted by dashed lines in Fig. 4) shows a shift in the onset and the maximum of the growth rate of the KBM to lower $\beta_{e}$ region. However, for this case, the experimental value of $\beta_{e}=0.0016$ is also shifted towards lower $\beta_{e}$, and therefore, experimentally, the plasma is well inside the ITG mode dominated region. There is a slight increase in both growth rate and real frequency of the ITG mode from case 1 to case 2 , for the experimentally relevant $\beta_{e}$ domain.

The $\beta_{e}$ dependence of the nickel peaking factor is interesting, since for the ITG branch it shows an increase with
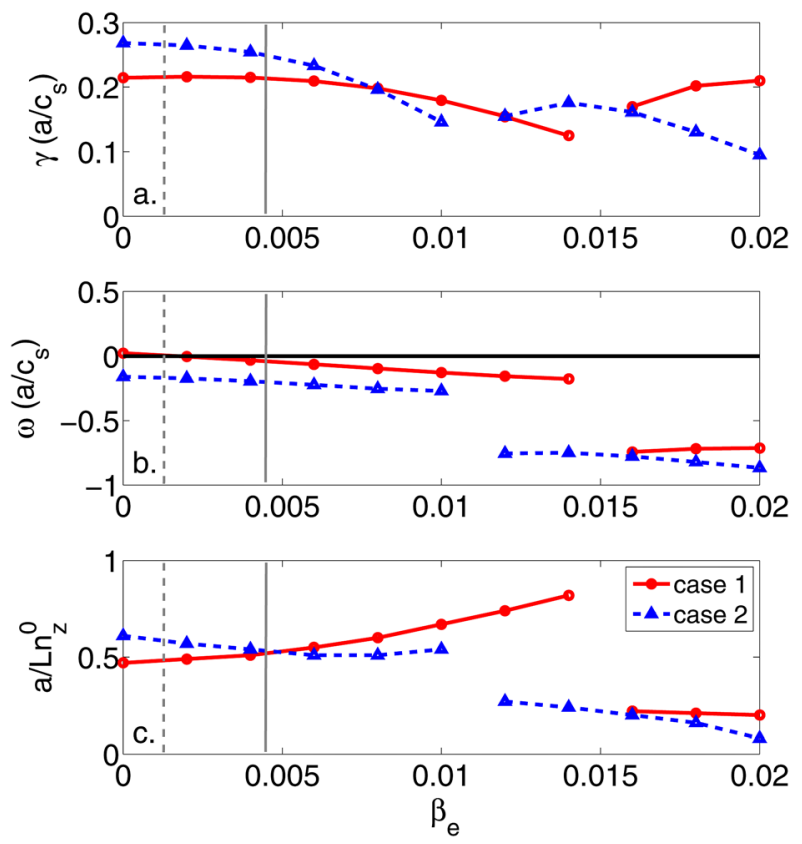

FIG. 4. (Color online) (a) Imaginary and (b) real parts of the eigenfrequency $\omega+i \gamma$ as function of $\beta_{e}$ obtained by GYRO for the baseline cases 1 and 2 . Solid lines (red with circle markers) represent case 1, dashed lines (blue with triangle markers) correspond to case 2 . The frequencies are normalized to $c_{s} / a$. (c) Peaking factor for nickel as a function of $\beta_{e}$ for cases 1 and 2 . The vertical solid and dashed lines represent the experimental value of $\beta_{e}$ for case 1 and 2, respectively.

increasing $\beta_{e}$, while in the $\mathrm{KBM}$ branch, the values are significantly lower (about half of that in the ITG branch) with no strong dependence on $\beta_{e}$. This is because in a linear model, the level of transport is inversely proportional to the ratio of the real frequency to the growth rate which is higher in the KBM branch, and this leads to a lower level of transport. The same qualitative behavior is present in both cases 1 and 2; however at outer toroidal flux $\rho_{\text {tor }}=0.55$ (case 2) in the ITG branch, the peaking factor has weaker $\beta_{e}$ dependence, and in the experimentally relevant $\beta_{e}$ region, the peaking factors are comparable to those corresponding to the lower radius $\rho_{\text {tor }}=0.34$ (case 1 ). No sign change for the peaking factor has been observed in our results. Other mechanisms can be responsible for the sign change of the impurity peaking factor such as the Ware pinch or RF induced poloidal asymmetries of the impurity density which can result in negative peaking factors for certain plasma conditions. ${ }^{21,22}$

Figure 5 shows the diffusivity $\left(D_{z}\right)$ and convective velocity $\left(a V_{z}\right)$ for the nickel impurity as a function of $\beta_{e}$ for the baseline cases 1 . These values are calculated using a linear fit for the impurity particle flux $\Gamma_{z}\left(=-n_{z} D / L_{n z}+n_{z} V_{z}\right)$ obtained by GYRO, as a function of impurity density gradient scale length $a / L_{n z}$. Here, the slope of the linear fit will represent the diffusivity coefficient $D_{z}$ and the cross point with the $a / L_{n z}=0$ line will represent $a V_{z}$ values. As seen in this figure in the ITG branch, the diffusivity shows a stronger dependence on $\beta_{e}$, while the convection is less sensitive to $\beta_{e}$. In agreement with experiments ${ }^{12,13}$ and previous studies, ${ }^{14}$ we observe that with increase of $\beta_{e}$, the diffusivity strongly reduces, which is in direct relation to the stabilization of the ITG modes with increase of $\beta_{e}$, see solid lines in 


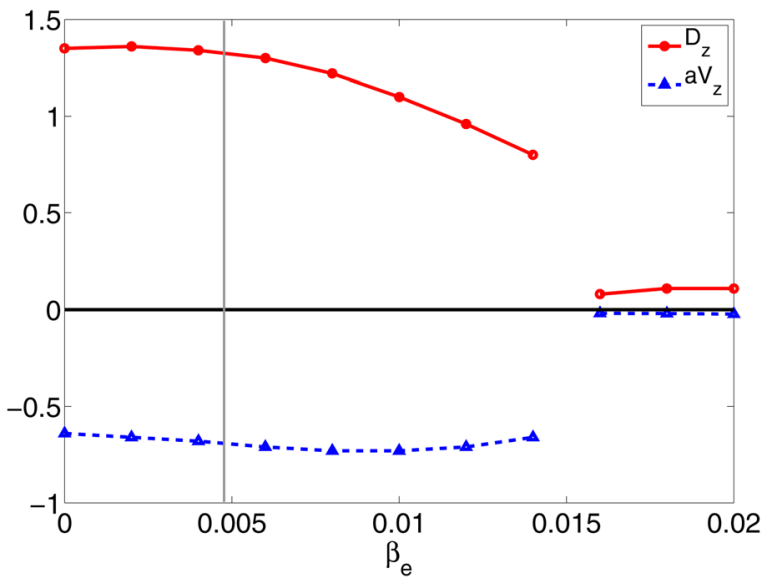

FIG. 5. (Color online) Diffusivity $\left(D_{z}\right)$ and convective velocity $\left(a V_{z}\right)-$ normalized to $a c_{s} k_{\theta} \rho_{s}\left|\phi_{n}\right|^{2}$ according to the notation of Ref. 17 - for nickel impurity as function of $\beta_{e}$ obtained by GYRO for the baseline case 1. Solid lines (red with circle markers) represent the diffusivity and dashed lines (blue with triangle markers) correspond to the minor radius times convective velocity. The vertical line represents the experimental value of $\beta_{e}$.

Fig. 4(a). Since the convective velocity shows no significant dependence on $\beta_{e}$, in combination with the decrease of diffusivity with $\beta_{e}$, the resulting peaking factor increases as $\beta_{e}$ increases, as seen in Fig. 4(c) (solid lines). In the KBM branch, however, both diffusivity and convective velocity show almost no dependence on $\beta_{e}$ and are significantly smaller than those in ITG branch.

\section{A. Charge dependence}

Figure 6 shows the peaking factors as function of $\beta_{e}$ for various impurity species in their fully ionized state (except for $\mathrm{W}$, where we assume $Z=40$ ). There is a different trend in the ITG and KBM branches as the impurity charge increases. In the electrostatic limit $\left(\beta_{e}=0\right)$, the ITG branch shows negligible charge dependence, however, as $\beta_{e}$ increases a nonnegligible dependence on the impurity charge appears in this branch with lower peaking factors for heavier impurities. The

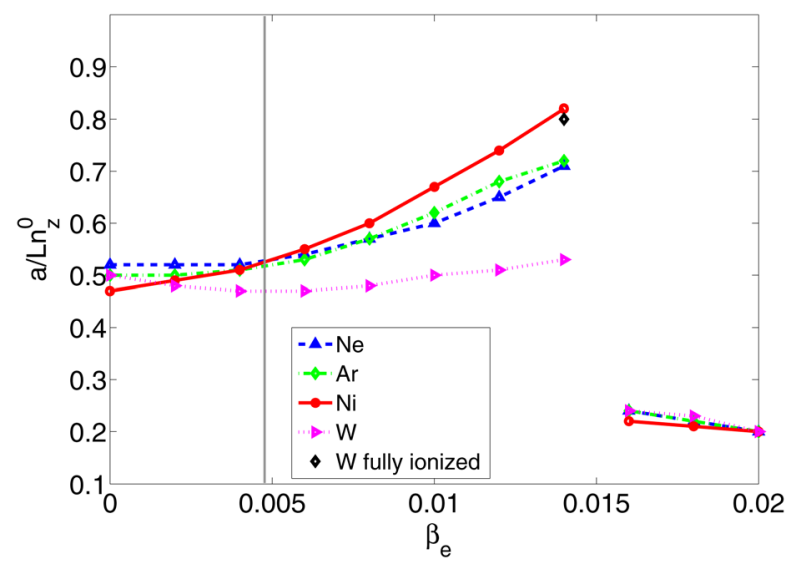

FIG. 6. (Color online) Peaking factor for different impurities as a function of $\beta_{e}$ for the baseline parameters case 1. Dashed line (blue) $\mathrm{Ne}^{+10}$, dasheddotted line (green) $\mathrm{Ar}^{+18}$, solid line (red) $\mathrm{Ni}^{+28}$, and dotted line (mauve) $\mathrm{W}^{+40}$. Black diamond represents the fully ionized tungsten $\mathrm{W}^{+74}$ at $\beta_{e}=0.014$. The vertical line represents the experimental value of $\beta_{e}$. peaking factor increases as $\beta_{e}$ increases except in the case of tungsten which shows almost no $\beta_{e}$ dependence. The reason for the difference in the behavior of tungsten is due to its lower charge to mass ratio $(\sim 1 / 4)$ compared to the other impurities, where this ratio is about a factor of two higher $(\sim 1 / 2)$. When we consider fully ionized tungsten $\mathrm{W}^{+74}$, a similar trend to that of the other impurities was observed (see the black diamond in Fig. 6). The parallel compressibility effects proportional to the charge to mass ratio, therefore, seem to play a crucial role in reducing the peaking factor for heavier impurities in the presence of finite $\beta_{e}$. This impact of charge to mass ratio can be beneficial for tokamaks with high$Z$ wall components like tungsten (it is usually not fully ionized in the core) for which a high $\beta$ operation with the ITG branch as the most unstable mode present can result in lower accumulation in the plasma core with almost no dependence on $\beta_{e}$. These results seem favorable for high $\beta$ plasma operations and are in agreement with previous results indicated by both experiments $^{2,12}$ and simulations ${ }^{6,9}$ that the particle and heat transport have very little or no dependence on $\beta$. In the KBM branch, no charge dependence is observed, and the peaking factors are about half of their values in the ITG branch for the whole considered range of $\beta_{e}$.

\section{B. The parametric dependences of the critical beta}

In order to examine the parametric dependence of the critical $\beta_{e}$ at which the ITG switches to KBM, we have performed a series of scans in various plasma parameters. Figures 7 to 9 present the results of these scans. Figures 7 and 8 show that as the gradients of the ion and electron temperatures or the electron density increase, the threshold moves towards lower $\beta_{e}$ values. Note that if the value of $a / L_{T i}$ is lower or the values of $a / L_{T e}$ or $a / L_{n e}$ are higher, the ITG mode at low $\beta_{e}$ can be converted to a TE mode, but even in this case, the KBM branch remains unstable at high $\beta_{e}$. This can be seen in the TE dominated cases shown in Figs. 7(a) and 7(c) by dashed curves corresponding to $a / L_{T i}=1.5$ and in Figs. 7(b) and 7(d) by dashed-dotted curves corresponding to $a / L_{T e}=3.0$, where mode frequencies are in the electron diamagnetic direction (positive). Also, shown in Figs. 8(a) and 8(b) by dashed-dotted curves corresponding to $a / L_{n e}=2.0$. This is consistent with previous observations that the $a / L_{T i}$ threshold for the KBM is lower than for the ITG mode. ${ }^{10}$ When the gradients are changed so that it is more favorable with TE-like behavior than ITG-like the peaking factors can decrease significantly, while the peaking factor in the KBM regime is almost completely insensitive to the change in these parameters.

From Fig. 9(a), we note that a 30\% variation in magnetic shear, $s$, does not seem to affect the $\beta_{e}^{\text {crit }}$, but from Fig. 9(b), it is clear that $\beta_{e}^{\text {crit }}$ is very sensitive to the safety factor $q$. As seen in this figure, $\beta_{e}^{\text {crit }}$ shifts towards lower $\beta_{e}$ values as $q$ increases. The reason for such a strong sensitivity can be understood through the stabilizing impact of the high $q$ on the ITG branch, see Refs. 4 and 23-25. With increasing $q$, the $\beta_{e}$ value for which the ITG mode becomes stable decreases, and so does the onset of KBM mode and $\beta_{e}$ for which the KBM branch becomes the dominant instability. 

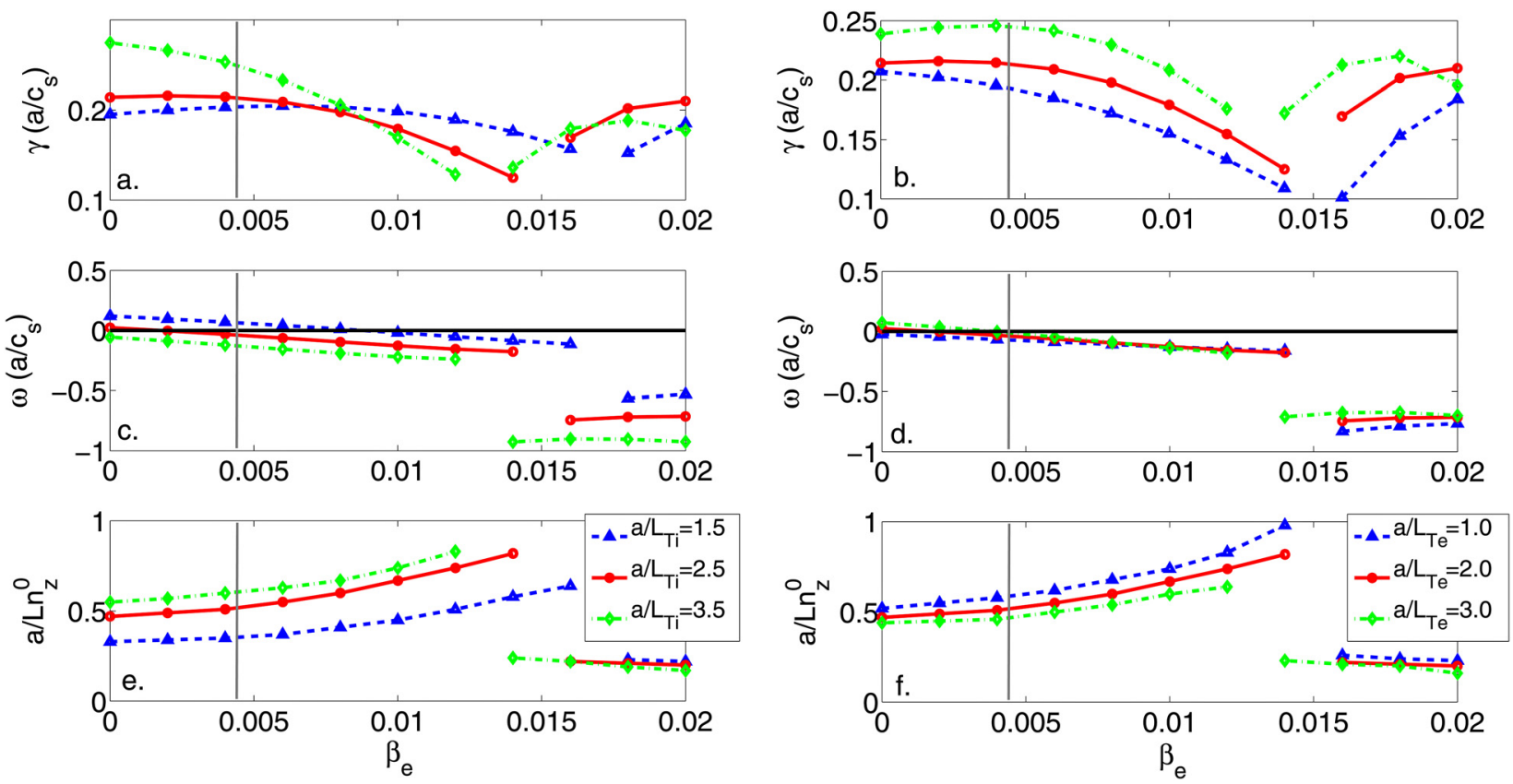

FIG. 7. (Color online) (a), (b) Imaginary and (c), (d) real parts of the eigenfrequency $\omega+i \gamma$ as function of $\beta_{e}$ obtained by GYRO for the baseline case 1 and two other $a / L_{T i}$ (a, c) and $a / L_{T e}$ (b, d) values. The frequencies are normalized to $c_{s} / a$. (e), (f) Peaking factor for nickel as a function of $\beta_{e}$ corresponding to eigenvalues in (a)-(d). Solid lines correspond to the case 1 parameters. In (a, c, e), dashed lines correspond to $a / L_{T i}=1.5$, and dash-dotted lines correspond to $a / L_{T i}=3.5$. In (b, d, f), dashed lines correspond to $a / L_{T e}=1.0$, and dashed-dotted lines correspond to $a / L_{T e}=3.0$. The vertical lines represent the experimental value of $\beta_{e}$.

As seen in Fig. 9(a), the growth rates increase strongly in the KBM branch as the shear $s$ increases, while in the ITG branch, no significant modification in the growth rates is observed. The nickel peaking factor increases slightly in
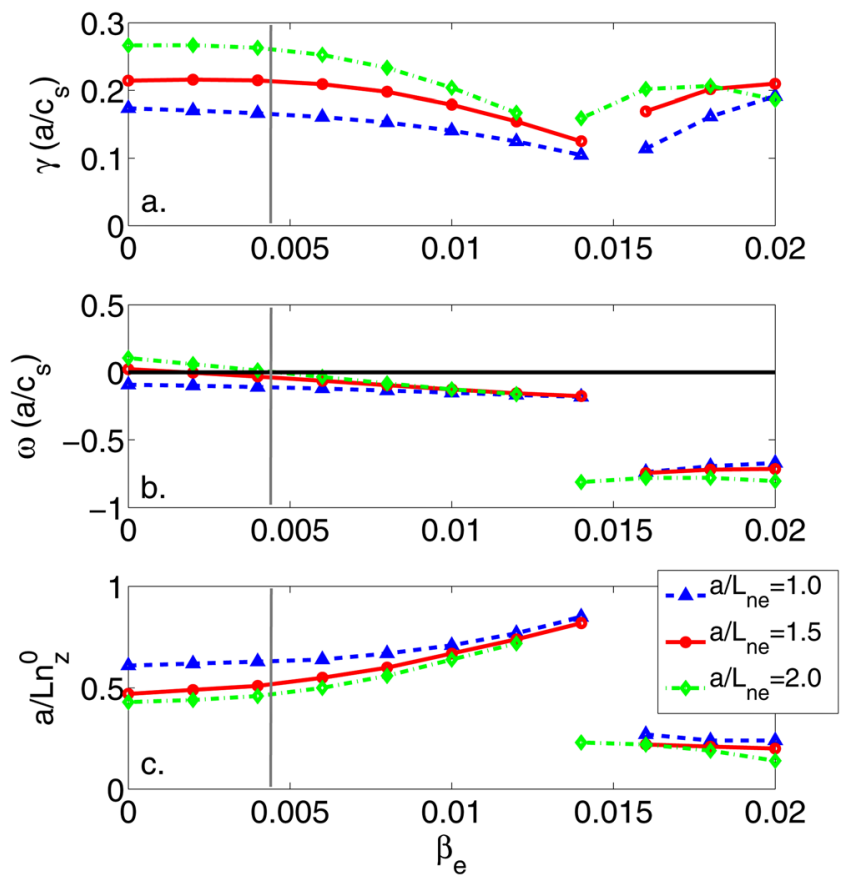

FIG. 8. (Color online) (a) Imaginary and (b) real parts of the eigenfrequency $\omega+i \gamma$ as function of $\beta_{e}$ obtained by GYRO for the baseline case 1 and two other $a / L_{n e}$ values. The frequencies are normalized to $c_{s} / a$. (c) Peaking factor for nickel as a function of $\beta_{e}$ corresponding to eigenvalues in (a) and (b). Solid lines correspond to the case 1 parameters, dashed lines correspond to $a / L_{n e}=1.0$, and dashed-dotted lines correspond to $a / L_{n e}=2.0$. The vertical lines represent the experimental value of $\beta_{e}$.
KBM branches as $s$ increases, see Fig. 9(e). No strong modifications are seen on the growth rates and the peaking factors as $q$ decreases, see Figs. 9(b) and 9(f).

For the experiment considered in our study, as seen in Figs. 7 to 9 , the impurity peaking factor in the KBM branch is about half of that in the ITG branch in all the scans, with very weak dependence on $\beta_{e}$. However, $\beta_{e}^{\text {crit }}$ for KBM onset is well above the experimental values, as given in Table II and therefore, the plasma is well inside the ITG branch with higher level of expected impurity accumulations.

\section{Compressional magnetic perturbations $\delta B$}

In most studies of gyrokinetic transport, only the transverse electromagnetic perturbations $\delta A_{\|}$have been retained and compressional magnetic perturbations $\delta B_{\|}$have been neglected. ${ }^{14}$ However, it has also been shown that in high $\beta$ plasmas, these effects can be large and neglecting them can lead to significant underestimating of the growth rates at high $\beta .^{4,7}$ In these references, it is argued that the so called "self-dug" well stabilizing impact on the $\nabla B$ drift is artificially overestimated if $\delta B_{\|}$is neglected for high $\beta$ analysis. Therefore, we examined the importance of these effects on the impurity peaking factors. Figure 10 shows the impact of $\delta B_{\|}$on the eigenvalues of the unstable modes and the impurity peaking factor. In agreement with previous works neglecting $\delta B_{\|}$will result in underestimating the growth rates but mainly for the KBM branch. In the absence of $\delta B_{\|}$ effects for the whole range of $\beta_{e}$ considered, no unstable $\mathrm{KBM}$ branch is observed and the ITG mode is the dominant instability. In this case, the impurity peaking factor also shows a very strong increase with increase of $\beta_{e}$ and, therefore, is strongly overestimated for high $\beta_{e}$ values. 

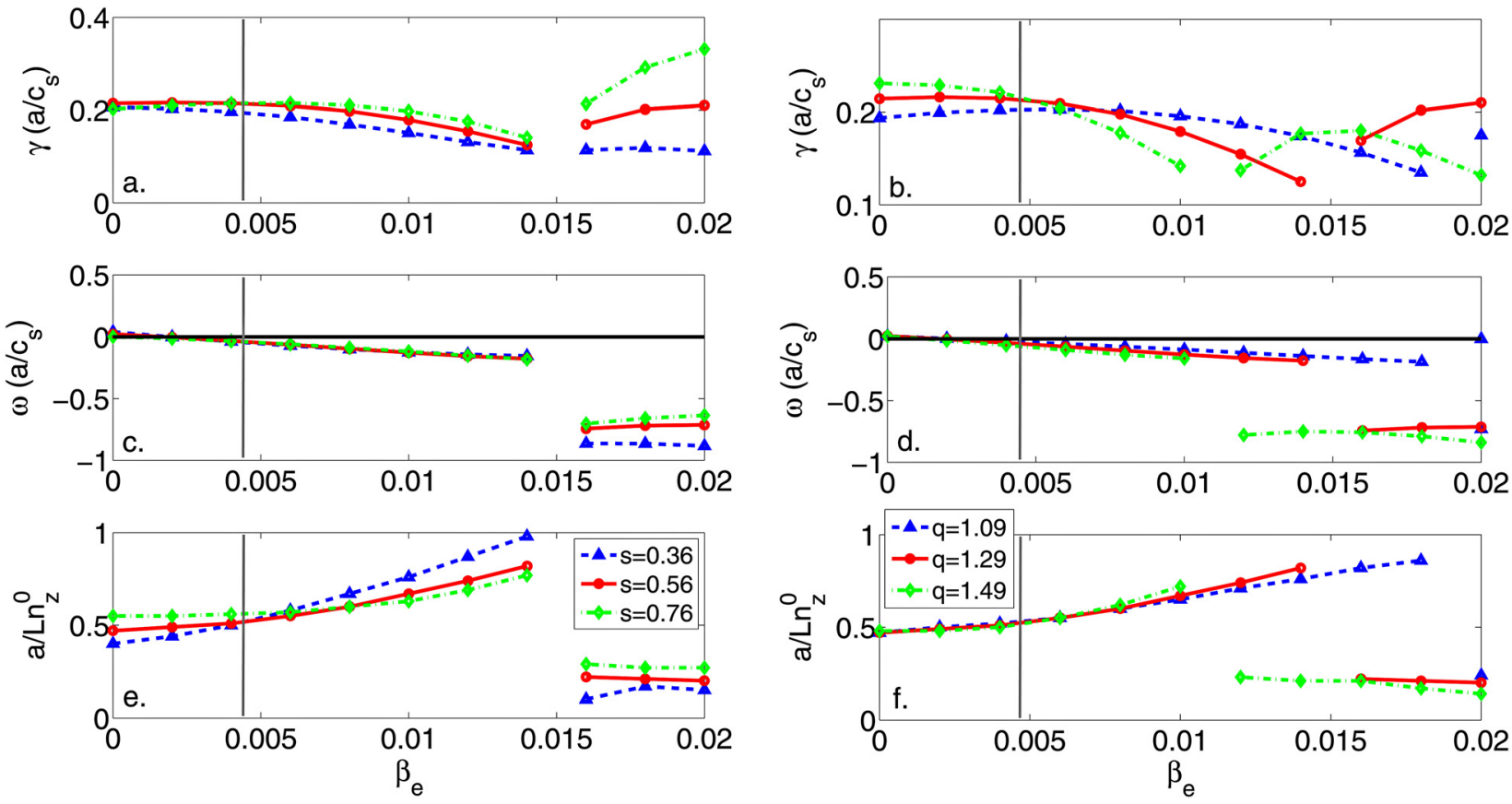

FIG. 9. (Color online) (a), (b) Imaginary and (c), (d) real parts of the eigenfrequency $\omega+i \gamma$ as function of $\beta_{e}$ obtained by GYRO for the baseline case 1 and two other $s(\mathrm{a}, \mathrm{c})$ and $q(\mathrm{~b}, \mathrm{~d})$ values. The frequencies are normalized to $c_{s} / a$. (e), (f) Peaking factor for nickel as a function of $\beta_{e}$ corresponding to eigenvalues in (a) and (b). Solid lines correspond to the case 1 parameters. In (a, c, e), dashed lines correspond to $s=0.36$, and dashed-dotted lines correspond to $s=0.76$. In (b, d, f), dashed lines correspond to $q=1.09$ and dashed-dotted lines correspond to $q=1.49$. The vertical lines represent the experimental value of $\beta_{\rho}$.

\section{Effect of MHD- $\alpha_{p}$ parameter}

Previous works have investigated the impact of $\alpha_{p}$ on microinstabilities. It has been shown that $\alpha_{p}$ can have stabilizing influence on drift waves in all branches, i.e., ITG mode, TE mode, electron temperature gradient (ETG) mode,
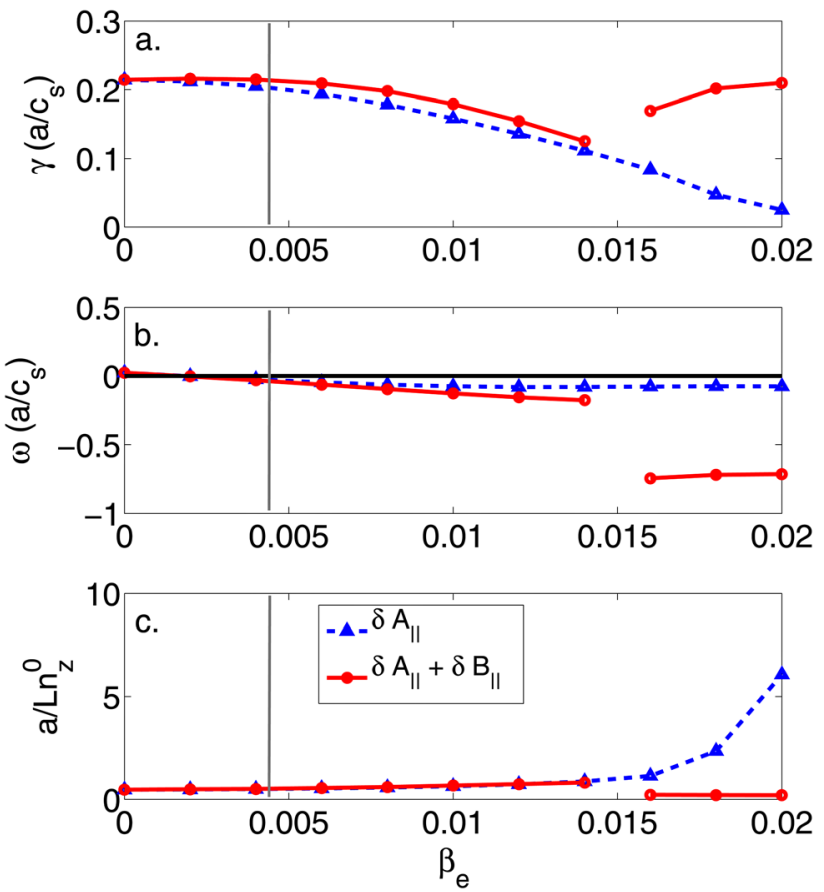

FIG. 10. (Color online) (a) Imaginary and (b) real parts of the eigenfrequency $\omega+i \gamma$ as function of $\beta_{e}$ obtained by GYRO for baseline case 1 with (solid lines) and without (dashed lines) $\delta B_{\|}$effects. The frequencies are normalized to $c_{s} / a$. (c) Peaking factor for nickel as a function of $\beta_{e}$ corresponding to eigenvalues in (a) and (b). The vertical lines represent the experimental value of $\beta_{e}$. and KBM. ${ }^{4,7,26}$ This effect is similar to the stabilizing effect of low or negative magnetic shear on the microinstabilities through significant reduction of the magnitude of the curvature and $\nabla B$ drifts. Therefore, $\alpha_{p}$ can be thought as acting as a local negative shear. Figure 11 shows the impact of $\alpha_{p}$ on the real and imaginary frequencies for the most unstable
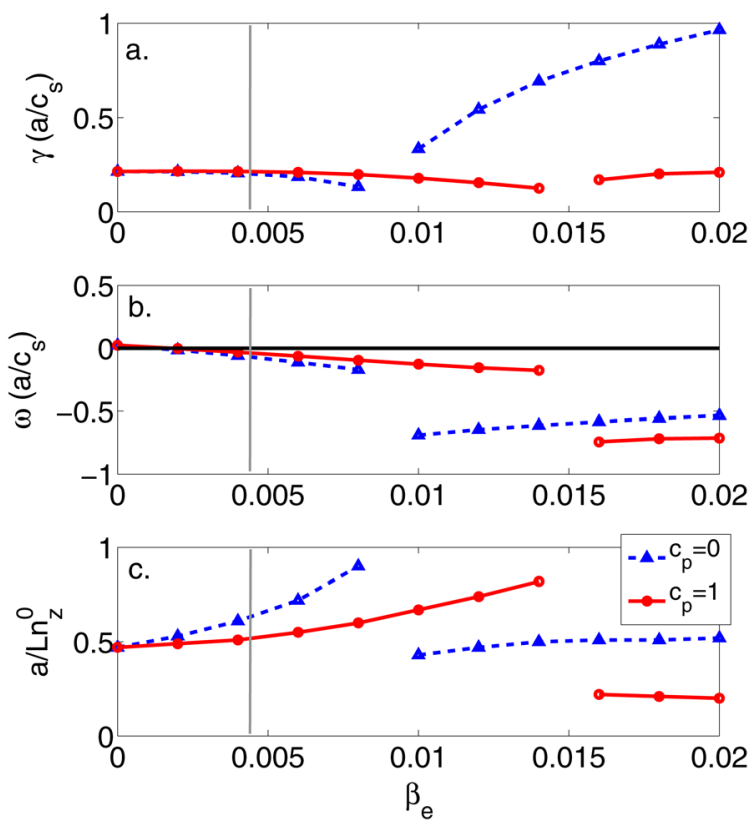

FIG. 11. (Color online) (a) Imaginary and (b) real parts of the eigenfrequency $\omega+i \gamma$ as function of $\beta_{e}$ obtained by GYRO for baseline case 1 with (solid lines) and without (dashed lines) MHD- $\alpha_{p}$ effects. The frequencies are normalized to $c_{s} / a$. (c) Peaking factor for nickel as a function of $\beta_{e}$ corresponding to eigenvalues in (a) and (b). The vertical lines represent the experimental value of $\beta_{e}$. 

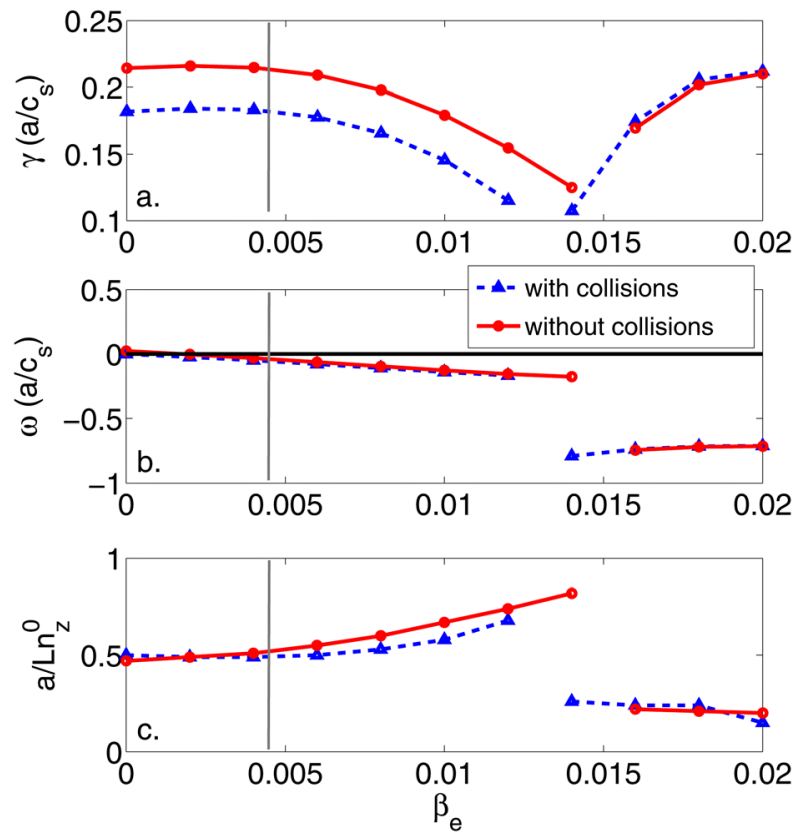

FIG. 12. (Color online) (a) Imaginary and (b) real parts of the eigenfrequency $\omega+i \gamma$ as function of $\beta_{e}$ obtained by GYRO for baseline case 1 with (solid lines) and without (dashed lines) collisions. The frequencies are normalized to $c_{s} / a$. (c) Peaking factor for nickel as a function of $\beta_{e}$ corresponding to eigenvalues in (a). The vertical lines represent the experimental value of $\beta_{e}$.

modes and on impurity peaking factor, where $\alpha_{p}$ varies from 0.076 in the ITG branch $\left(\beta_{e}=0.002\right)$ to 0.76 in the KBM branch $\left(\beta_{e}=0.02\right)$. As seen here by comparing the cases with (solid lines) and without (dashed lines) MHD- $\alpha$ effects, $\alpha_{p}$ has a significant stabilizing impact on the KBM growth rates, but interestingly, the ITG growth rates are even enhanced when the MHD- $\alpha$ is retained. However, $\alpha_{p}$ has the same effect regarding the peaking factors both in the ITG and the KBM regions; Fig. 11(c) shows a strong reduction in both ITG and KBM branches when $\alpha_{p}$ effects are included.

\section{E. Collisional effects}

Figure 12 shows the effects of collisions on the growth rate and real frequencies of the most unstable modes as well as on the peaking factor for nickel impurity. Here, the electron-ion and ion-ion collisions have been considered with $\nu_{e i}=0.0058$ in $c_{s} / a$ units (corresponding to $T_{e}=7 \mathrm{keV}$, $n_{i}=3 \times 10^{19} \mathrm{~m}^{-3}$, and $a=1 \mathrm{~m}$ ). As illustrated in Fig. 12(a) in the ITG branch, the growth rate is reduced with collisions, but in the KBM branch, there is almost no impact from collisions on the growth rate as was previously reported in Ref. 27. Also, as the ITG mode is stabilized further with collisions, the KBM threshold is shifted to lower $\beta_{e}$. However, Fig. 12(c) shows that a very small reduction of the impurity peaking factor is expected in the ITG branch due to collisions with almost no effect in the KBM branch.

\section{CONCLUSIONS}

We have investigated the impact of electromagnetic effects at finite $\beta$ on the transport of impurities and the onset of the KBM instability. The quasilinear impurity transport is computed using the gyrokinetic code GYRO in the flux-tube (local) limit. Two sets of parameters corresponding to two different radii ( $\rho_{\text {tor }}=0.34$ and 0.55 ) have been chosen from a recent experiment at JET with nickel laser blow off in $\mathrm{H}$ mode plasmas with $\beta_{e} \gtrsim 0.0016$ at the inner mid-radius. The transport of nickel impurities in these series of experiments has been discussed in Ref. 15, and it is shown that the peaking factor is reduced to almost zero at the mid-radius with a sign change from positive to negative for more inner radii. As shown in this work, the most unstable mode at these radial positions is the ITG mode, which is stabilized with increase of $\beta_{e}$. This stabilization is propagated through to the diffusivity coefficient, and in agreement with previous experimental and theoretical reports, we observe a strong reduction of the impurity diffusivity with increasing $\beta_{e}$. The convective velocity, on the other hand, is almost insensitive to $\beta_{e}$. We found that for the experimentally relevant $\beta_{e}$, comparable peaking factors are expected for both radii. No sign change for the peaking factor has been observed in our results. Other effects that we have not considered here (Ware pinch and poloidal asymmetries) may be a part of the explanation for the sign change. In the KBM branch, we observe that diffusivity and convective velocity are both almost independent of $\beta_{e}$, and in comparison with their values in ITG branch, a strong reduction is obtained. The corresponding peaking factor is also strongly reduced and is only very weakly dependent on $\beta_{e}$.

The $\beta_{e}^{\text {crit }}$ for the onset of the KBM is found to be around $\beta_{e} \sim 0.01$, and as the ITG branch is stabilized with increasing $\beta_{e}$, the $\mathrm{KBM}$ branch becomes the dominant instability at around $\beta_{e} \sim 0.014$. For the experiment considered here, this threshold is sensitive to safety factor $q$ and gradients of density and temperatures. For high $q$, the threshold moves towards lower $\beta_{e}$. This shift is due to stabilization of the ITG branch with higher $q$, resulting in the KBM branch to become the dominant mode at lower $\beta_{e}$. It is observed here that the $a / L_{T i}$ threshold for the onset of KBMs is lower than that for the ITG modes. Effects of compressional magnetic perturbations $\delta B_{\|}$, collisionality, and MHD- $\alpha_{p}$ parameter on the $\beta_{e}^{\text {crit }}$ are investigated. It is found that these effects have a strong impact on the characteristics of the unstable modes and the onset of KBM instability and neglecting them can lead to misleading results specially at high $\beta_{e}$. It was found that without $\delta B_{\|}$effects, the KBM threshold is overestimated and that the $\alpha_{p}$ has a significant stabilizing impact on the growth rates of the unstable modes as $\beta_{e}$ is increased. This stabilizing impact is propagated through to the impurity peaking factor with a strong reduction in the KBM branch when MHD- $\alpha_{p}$ effects are included. For the ITG branch, the effect is negligible. In contrast, the impact of collisions is mostly visible on the ITG branch, and it leads to a reduction in the growth rate of the unstable mode and peaking factor.

The charge dependence of the peaking factor was analyzed and it was found that in the electrostatic limit $\left(\beta_{e}=0\right)$, the ITG branch shows negligible charge dependence; however as $\beta_{e}$ increases, a non-negligible dependence on the impurity charge appears increasing with $\beta_{e}$, except in the case of tungsten which shows lower peaking factors with almost no dependence on $\beta_{e}$. The parallel compressibility effects proportional to the charge to mass ratio seem to play a 
crucial role in reducing the peaking factor for heavier impurities in the presence of finite $\beta_{e}$ effects. This impact of charge to mass ratio can be beneficial for tokamaks with high- $Z$ wall components like tungsten for which lower accumulations in the core can be possible in high $\beta$ plasma operations. In the KBM branch, no charge dependence is observed.

In conclusion, in the core of $\mathrm{H}$-mode plasmas where $\beta_{e}$ can be high enough for the ITG modes to be strongly stabilized, the impurity diffusivity is reduced. However, since the impurity convective velocity is almost independent of $\beta_{e}$, the corresponding peaking factor increases with $\beta_{e}$. For not fully ionized heavy impurities like tungsten, however, lower peaking factors and no dependence on $\beta_{e}$ is expected. In the view of the new all tungsten divertor at the JET tokamak, $\beta$ scaling experiments are needed in order to confirm these results. The KBM instability threshold can be much lower than the ideal MHD threshold depending on the plasma parameters and particularly strongly on plasma shape. Due to the importance of the magnetic geometry, the model geometry used in the gyrokinetic simulations can affect the results significantly, and the commonly used $s$ - $\alpha$ model overestimates the $\mathrm{KBM}$ growth rates and ITG stabilization at high $\beta$.

\section{ACKNOWLEDGMENTS}

The authors would like to thank J. Candy for the valuable comments and for providing the GYRO code. This work was funded by the European Communities under Association Contract between EURATOM and Vetenskapsraidet. The views and opinions expressed herein do not necessarily reflect those of the European Commission.

\footnotetext{
${ }^{1}$ M. Shimada, D. J. Campbell, V. Mukhovatov, M. Fujiwara, N. Kirneva, K. Lackner, M. Nagami, V. D. Pustovitov, N. Uckan, and J. Wesley International Tokamak Physics Activity Topical Group Chairs, Cochairs and Chapter Coordinators: N. Asakura, A. E. Costley, A. J. H. Donné, E. J. Doyle, A. Fasoli, C. Gormezano, Y. Gribov, O. Gruber, T. C. Hender, W. Houlberg, S. Ide, Y. Kamada, A. Leonard, B. Lipschultz, A. Loarte, K. Miyamoto, V. Mukhovatov, T. H. Osborne, A. Polevoi, and A. C. C. Sips, Nucl. Fusion 47, S1 (2007).
}

${ }^{2}$ D. C. McDonald, L. Laborde, J. C. DeBoo, F. Ryter, M. Brix, C. D. Challis, P. de Vries, C. Giroud, J. Hobrik, D. Howell, E. Joffrin, T. C. Luce, J. Mailloux, V. Pericoli-Ridolfini, A. C. C. Sips, K. Thomsen, and JET EFDA Contributors, Plasma Phys. Controlled Fusion 50, 124013 (2008).

${ }^{3}$ P. B. Snyder and G. W. Hammett, Phys. Plasmas 8, 744 (2001).

${ }^{4}$ C. Bourdelle, W. Dorland, X. Garbet, G. W. Hammett, M. Kotschenreuther, G. Rewoldt, and E. J. Synakowski, Phys. Plasmas 7, 2881 (2003).

${ }^{5}$ J. Y. Kim, W. Horton, and J. Q. Dong, Phys. Fluids B 5, 4030 (1993).

${ }^{6}$ J. Candy, Phys. Plasmas 12, 072307 (2005).

${ }^{7}$ E. A. Belli and J. Candy, Phys. Plasmas 17, 112314 (2010).

${ }^{8}$ F. Jenko and W. Dorland, Plasma Phys. Controlled Fusion 43, A141 (2001).

${ }^{9}$ M. J. Pueschel, M. Kammerer, and F. Jenko, Phys. Plasmas 15, 102310 (2008).

${ }^{10} \mathrm{G}$. Zhao and L. Chen, Phys. Plasmas 9, 861 (2002).

${ }^{11}$ J. Dong, Nucl. Fusion 39, 1041 (1999).

${ }^{12}$ C. C. Petty, T. C. Luce, D. C. McDonald, J. Mandrekas, M. R. Wade, J. Candy, J. G. Cordey, V. Drozdov, T. E. Evans, J. R. Ferron, R. J. Groebner, A. W. Hyatt, G. L. Jackson, R. J. La Haye, T. H. Osborne, and R. E. Waltz, Phys. Plasmas 11, 2514 (2004).

${ }^{13}$ K.-D. Zastrow, J. M. Adams, Yu Baranov, P. Belo, L. Bertalot, J. H. Brzozowski, C. D. Challis, S. Conroy, M. de Baar, P. de Vries, P. Dumortier, J. Ferreira, L. Garzotti, T. C. Hender, E. Joffrin, V. Kiptily, J. Mailloux, D. C. McDonald, R. Neu, M. O’Mullane, M. F. F. Nave, J. Ongena, S. Popovichev, M. Stamp, J. Stober, D. Stork, I. Voitsekhovitch, M. Valovič, H. Weisen, A. D. Whiteford, A. Zabolotsky, and JET EFDA Contributors, Plasma Phys. Controlled Fusion 46, B255 (2004).

${ }^{14}$ T. Hein, C. Angioni, E. Fable, and J. Candy, Phys. Plasmas 17, 102309 (2010).

${ }^{15}$ M. Valisa, L. Carraro, I. Predebon, M. E. Puiatti, C. Angioni, I. Coffey, C. Giroud, L. Lauro Taroni, B. Alper, M. Baruzzo, P. Belo daSilva, P. Buratti, L. Garzotti, D. Van Eester, E. Lerche, P. Mantica, V. Naulin, T. Tala, M. Tsalas, and JET-EFDA contributors, Nucl. Fusion 51, 033002 (2011).

${ }^{16}$ J. Candy and R. E. Waltz, J. Comput. Phys. 186, 545 (2003).

${ }^{17}$ J. Candy and E. Belli, General Atomics Report No. GA-A26818, 2011.

${ }^{18}$ C. F. Maggi, R. J. Groebner, C. Angioni, T. Hein, L. D. Horton, C. Konz, A. W. Leonard, C. C. Petty, A. C. C. Sips, P. B. Snyder, J. Candy, R. E. Waltz, ASDEX Upgrade, and DIII-D teams, Nucl. Fusion 50, 025023 (2010).

${ }^{19}$ R. E. Waltz and R. L. Miller, Phys. Plasmas 6, 4265 (1999).

${ }^{20}$ J. Candy, Plasma Phys. Controlled Fusion 51, 105009 (2009).

${ }^{21}$ T. Fülöp and S. Moradi, Phys. Plasmas 18, 030703 (2011).

${ }^{22}$ S. Moradi, T. Fülöp, A. Mollén, and I. Pusztai, Plasma Phys. Controlled Fusion 53, 115008 (2011).

${ }^{23}$ K. H. Burrell, Phys. Plasmas 4, 1499 (1997).

${ }^{24}$ X. Garbet, C. Bourdelle, G. T. Hoang, P. Maget, S. Benkadda, P. Beyer, C. Figarella, I. Voitsekovitch, O. Agullo, and N. Bian, Phys. Plasmas 8, 2793 (2011)

${ }^{25}$ J. E. Kinsey, R. E. Waltz, and J. Candy, Phys. Plasmas 13, 022305 (2006).

${ }^{26}$ C. Bourdelle, Nucl. Fusion 45, 110 (2005).

${ }^{27}$ G. Rewoldt, W. M. Tang, and R. J. Hastie, Phys. Fluids 30, 807 (1987). 